\title{
Nota del editor: Oportunidades y desafíos para la alfabetización mediática informacional
}

\author{
Editor's note: Opportunities and challenges for media and \\ information literacy
}

Andrea De-Santis

Universidad Politécnica Salesiana del Ecuador y Universitat Autónoma de Barcelona

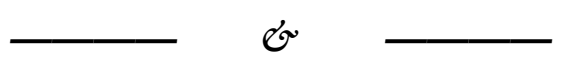

\section{NOTA DEL EDITOR: OPORTUNIDADES Y DESAFÍOS PARA LA ALFABETIZACIÓN MEDIÁTICA INFORMACIONAL}

\begin{abstract}
Resumen: En el nuevo contexto social determinado por el desarrollo contante de las TIC, los ciudadanos ejercen su rol activo mediante dispositivos tecnológicos, y la alfabetización mediática se presenta como un agente de cambio en favor de una sociedad más crítica, democrática y participativa. En esta edición especial se presentan seis contribuciones que aportan al debate sobre los retos de la alfabetización mediática en el siglo XXI mediante el estudio de fenómenos y aspectos en los cuales el uso de las TIC y los medios digitales convergen con la necesidad de ofrecer propuestas educativas que respondan a las exigencias formativas de la ciudadanía. Los autores otorgan miradas profundas sobre cuestiones como el acceso a la educación y la tecnología, la interacción lúdica en la educación, la motivación de los educandos en su participación, y la materialización de estas reflexiones en propuestas educativas de cuarto nivel.
\end{abstract}

Palabras clave: Alfabetización mediática informacional; Medios digitales; Educación; Aprendizaje digital 


\title{
EDITOR'S NOTE: OPPORTUNITIES AND CHALLENGES FOR MEDIA AND INFORMATION LITERACY
}

\begin{abstract}
In the new social context determined by the constant development of ICT, citizens exercise their active role through technological devices, and media literacy is presented as an agent of change in favour of a more critical, democratic and participatory society. In this special edition, six papers are presented to contribute to the discussion on the challenges of media literacy in the 21st century through the study of phenomena and aspects in which the use of ICT and digital media converge with the need to offer educational proposals that respond to the citizen's formative demands. The authors give deep insights into issues such as access to education and technology, playful interaction in education, learners' motivation in their participation, and the materialisation of these reflections into fourth level educational proposals.
\end{abstract}

Keywords: Media literacy; Digital media; Education; Digital learning

Desde la idealización de la Aldea Global de McLuhan $(1962 ; 1964)$ asistimos a la configuración de un nuevo contexto social marcado por el rápido y dinámico desarrollo de las tecnologías de la información, la comunicación y los medios digitales, en el cual las personas asumen el rol de prosumidores usando las TIC para materializar su rol activo de ciudadanos. Los usuarios se conectan en redes participando en un ecosistema multiplataforma que favorece la interacción social, el cual se sostiene en buena parte sobre el uso de los dispositivos móviles (De Santis-Piras y Morales Morante, 2019). Ante la diversificación del contexto, el profesor Guillermo Orozco, en las páginas de este monográfico, sugiere que la alfabetización mediática se presenta como agente de cambio en favor de una sociedad más crítica, democrática y preparada para participar de un mundo globalizado. Al fin y al cabo, la comunicación se ha vuelto un eje central de esta transformación que ha evidenciado la necesidad imperante de formar ciudadanos con habilidades, competencias y actitudes que favorezcan su convivencia y participación en la sociedad digital y del conocimiento (Pérez-Tornero, 2017; Ruiz y Pérez-Escoda, 2020). La UNESCO reconoce que la Alfabetización Mediática e Informacional (MIL Media and Information Literacy) concierne a las funciones de los medios y las fuentes de información en nuestra vida personal y en las sociedades democráticas (UNESCO, 2011) promoviendo el derecho del individuo a comunicarse, expresarse, buscar, recibir e impartir información e ideas, fomentando la evaluación de los medios y las fuentes de información en función de cómo se producen, los mensajes que transmiten y la audiencia a la que se dirigen (UNESCO, 2011). Este último punto, sobre todo, abre un abanico de posibilidades de estudio y de enfoques disciplinarios para analizar y alcanzar importantes conclusiones sobre la comunicación multimedia y los múltiples ámbitos del aprendizaje. 
En esta edición especial de Bellaterra Journal of Teaching \& Learning Language \& Literature se presentan seis contribuciones de diez investigadores pertenecientes a siete universidades de Argentina, Ecuador y España. Aportan al debate sobre los retos de la alfabetización mediática en el siglo XXI mediante la investigación de fenómenos, proyectos y experiencias en las cuales el uso de las TIC y los medios digitales convergen con la necesidad de diseñar, organizar, desarrollar y evaluar propuestas educativas capaces de responder a las exigencias de formación de la ciudadanía como de su mayor, más activa y consciente participación en sociedades democráticas. Los autores de los trabajos de este número ofrecen miradas profundas sobre las cuestiones más relevantes de la alfabetización mediática, contribuyendo de manera significativa al estudio de aspectos como el acceso a la educación y la tecnología, la motivación de los educandos en su participación de programas formativos, y la materialización de estas reflexiones en propuestas educativas de cuarto nivel.

El primer artículo "Interacción lúdica hacia la educación en medios. Revisión sistemática de literatura científica" (Torres-Toukoumidis, Marín-Gutiérrez y De-Santis, Universidad Politécnica Salesiana y Universidad Técnica Particular de Loja, Ecuador) es una revisión sistemática de la literatura científica sobre la relación entre alfabetización mediática y juegos. Los investigadores presentan un análisis de la implicación teórica en la comunidad académica de las principales tendencias lúdicas aplicadas al ámbito de la educación, los juegos serios, de simulación y la gamificación. Los estudiantes del siglo XXI usan Internet como herramienta de aprendizaje y su proceso de socialización se materializa en los nuevos medios y mediante ellos, incluidos los videojuegos. Los autores muestran que la incorporación de las experiencias lúdicas en la alfabetización mediática se genera principalmente en la educación formal, donde se logra promover actividades colaborativas que repercuten en el desarrollo creativo de los estudiantes.

A continuación, se presentan tres artículos de investigación de estudiantes de doctorado, los cuales se caracterizan por la diversidad de puntos de vista. La primera de estas contribuciones, "La interculturalidad y las TIC's en la formación de posgrados en educación” (Tubay, UNED-Universidad Nacional de Educación a Distancia, España, y Universidad Técnica de Manabí, Ecuador), propone un estudio exploratorio sobre la oferta de programas de posgrado en educación en Ecuador y su construcción curricular desde los estudios interculturales, las tecnológicas educativas y las relaciones socioculturales. El objetivo es analizar la valoración de elementos documentales relacionados con la formación en el uso de las TIC y su efectiva presencia en la propuesta curricular. Su conclusión evidencia importantes 
vacíos curriculares en los cuales lo intercultural y lo tecnológico no constituyen aún en estas propuestas educativas elementos emergentes y transversales.

En "Incidencia del diseño instruccional y elementos alternativos en el engagement de participantes en MOOCs" (Castillo-Abdul, Bonilla-del-Río y Civila de Dios, Universidad de Huelva, España) las autoras identifican las características que influyen en la motivación de los participantes de tres reconocidas plataformas de cursos online, focalizando en los MOOC (Massive Open Online Courses) como una de las herramientas utilizadas en el ámbito de la educación la cual atrae mayor interés de la comunidad académica y científica. Las dificultades de acceso, la desactualización de las competencias mediáticas y, sobre todo, el diseño basado en una metodología tradicional constituyen los principales aspectos a tener en cuenta frente al desafío de motivar de los estudiantes a un mayor uso y participación mediante la TIC en favor de un proceso de aprendizaje efectivo.

El último artículo de investigación "Traspasar las pantallas: la interacción para el aprendizaje en entornos virtuales" (Ladaga, Universidad Nacional de La Plata, Argentina) presenta un estudio cualitativo de carácter interpretativo consistente en visualizar algunas de las condicionantes propias de la educación mediada por las tecnologías digitales, vinculadas a los procesos relacionales, las prácticas de uso y las lógicas de interacción del entorno virtual. El artículo contribuye de manera significativa a la controversia entre los sistemas de gestión de aprendizaje y el entorno personal de aprendizaje sugiriendo una convergencia de ambos en la propuesta pedagógico-comunicacional de aprendizaje en línea. Las conclusiones que nos ofrece la autora apuntan a la demanda de un sistema de educación en línea que otorgue más valor a los aspectos transmediales de la comunicación en los programas formativos.

Para entender de manera profunda la alfabetización mediática, sus efectos y su inserción dentro y fuera de las aulas de clases, presentamos una entrevista al Dr. Guillermo Orozco ("La alfabetización mediática e informacional injerencia y perspectiva en América Latina”, a cargo de González-Carrión, Universidad Nacional de Loja, Ecuador). El profesor Orozco, reconocido académico mexicano, ha contribuido de manera significativa a los estudios sobre audiencias, alfabetización audiovisual, producción, mediación y estrategias pedagógicas de intervención con los medios de comunicación. En la entrevista realiza un recorrido histórico de la conceptualización de la alfabetización mediática con enfoque en el contexto latinoamericano, evidenciando la importante contribución de la UNESCO en el reconocimiento y promoción de esas competencias, que desde su punto de vista deben ser 
consideradas dentro de las propuestas educativa actuales de manera categórica. Además, enfatiza sobre el rol de las instituciones públicas responsables de la educación insistiendo de manera contundente en su rol de promotores de la alfabetización mediática, así como las ventajas que esta trae para la Universidad y los investigadores favoreciendo su trabajo de comunicación académica formal, informal y científica.

La última contribución de este volumen es la reseña de la obra del lingüista Xavier Laborda Gil Cuadernos para el análisis 50. Casos ejemplares de comunicación. Comentarios y actividades para crecer hacia adentro (Guajardo Figueroa, Universidad Autónoma de Barcelona, España). Mediante la revisión y explicación de los cinco casos de comunicación propuestos por Laborda Gil, la reseña presenta el análisis del discurso y sus técnicas con particular atención a la capacidad de repercutir en otras personas mediante su uso. La investigadora enfatiza el propósito reflexivo del libro, destacando cómo su autor invita a usar la comunicación para crecer interna y externamente, a transformarse en seres pensantes con ambición de conocimiento para construir discursos que sean significativos para el receptor.

Al tratar los retos de la alfabetización mediática e informacional, esta edición especial ofrece una visión enriquecedora en mérito a las oportunidades educativas, participativas y tecnológicas que ofrece a la sociedad y a las dificultades que enfrenta, para las cuales se proponen estrategias y soluciones. A manera de conclusión, se ofrecen los principales desafíos identificados y definidos por el trabajo de investigación y análisis de los autores: a) incluir la alfabetización mediática en los currículos desde la educación básica; b) transformar la infraestructura tecnológica; c) estimular la participación, motivación y continuidad de los estudiantes en las propuestas educativas digitales; d) mantener una actualización constante de la formación, en particular de los profesores; y e) diseñar metodologías innovadoras y dinámicas colaborativas que integren de manera adecuada la tecnología.

\section{AgradeCimientos}

Como editor invitado quiero agradecer de manera especial las personas que hicieron posible la publicación de esta edición especial de Bellaterra Journal of Teaching \& Learning Language \& Literature iniciando por los mentores Dr. Xavier Fontich, co-editor de la revista, por la oportunidad y apoyo constante en el desarrollo del trabajo, y Dr. Luis Fernando Morales Morante, profesor de la UAB y director de tesis, por guiarme en el mundo de la investigación y la comunicación académica. 
Muchas gracias al Dr. Ángel Torres-Toukoumidis por el apoyo brindado y el contagioso entusiasmo de investigador. Un agradecimiento especial para los autores de los trabajos científicos y los evaluadores por hacer posible la realización de este número gracias a su profesionalismo, compromiso y amor para la ciencia.

\section{REFERENCES}

De Santis-Piras A., y Morales Morante, L. (2019). Dispositivos móviles y las multiplataforma de interacción lúdica. En A. Torres Toukoumidis, L. Romero-Rodríguez y J. Salgado Guerrero (Eds.), Juegos y Sociedad: desde la interacción a la inmersión para el cambio social (pp. 69-78). McGraw Hill.

McLuhan, M. (1962). The Gutenber Galaxy. Routledge.

McLuhan, M. (1964). Understanding Media: The Extensions of Man. McGraw Hill.

Pérez Tornero, J.M. (2017). Aprender valores con internet. Cómo potenciar la ética, el respeto, la tolerancia y la cooperación en internet. Octaedro.

Ruiz, R. y Pérez-Escoda, A. (2020). Comunicación y Educación en un mundo digital y conectado. Presentación, Icono 14, 18(2), 1-15. doi: https://doi.org/10.7195/ri14.v18i2.1580

UNESCO. (2011). Media and information literacy curriculum for teachers. Recuperado de bit.ly/32HqX1R

\section{ANDREA DE-SANTIS}

Profesor titular y director de los programas de Comunicación y Comunicación Social de la Universidad Politécnica Salesiana (Ecuador). Doctorando en Comunicación Estratégica, Publicidad y Relaciones Publicas por la UAB (España). Magister en Dirección de Comunicación por la UDLA (Ecuador), Dottore in Scienze della Comunicazione por La Sapienza Universitá di Roma (Italia).

\section{adesantis@ups.edu.ec https://orcid.org/0000-0002-7441-3794}

De-Santis, A. (2021). Nota del editor: Oportunidades y desafíos para la alfabetización mediática informacional. Bellaterra Journal of Teaching \& Learning Language \& Literature, 14(3), e982. https://doi.org/10.5565/rev/jtl3.951 